\title{
Generalidades del recurso hidrobiológico para la producción de la industria pesquera peruana*
}

\author{
Fernando Kleeberg Hidalgo, Mario Rojas Delgado \\ Universidad de Lima \\ Ingeniería Industrial n. ${ }^{\circ} 29,2011$, ISSN 1025-9929, pp. 51-72 \\ Recibido: 26 de abril del 2011 / Aprobado: 12 de julio del 2011
}

\begin{abstract}
Resumen: Se han identificado los factores de mayor incidencia en la evolución del recurso hidrobiológico para la producción de la industria pesquera peruana, mediante una investigación de tipo descriptivo-aplicativa, orientada al estudio del desarrollo del sector pesquero nacional. En la metodología se combina el trabajo de gabinete para la obtención y revisión de la información, con el trabajo de campo para su contrastación y validación. Se han considerado las generalidades sobre la materia prima como: las especies y caracterización, embarcaciones empleadas, las estadísticas y el desembarque potencial.
\end{abstract}

Palabras clave: recursos hidrobiológicos / industria pesquera / acuicultura

\section{Generalities of the hydrobiological resource for the production of the peruvian fishery industry}

\begin{abstract}
AвstRact: It has been identified the major incidental factors in the evolution of the hydrobiological resource for the production in the peruvian fishery industry, by means of a descriptive-applicative type investigation oriented toward the study of the national fishery sector development. The methodology combines the cabinet study to obtain and review information and a field work for contrast and validity. It has been considered generalities of raw material such as: characterization of species, boats being used, statistics and potential unloading.
\end{abstract}

Keywords: hydrobiological resources / fishing industry / aquiculture

* Este trabajo forma parte de la investigación sobre la industria pesquera en el Perú que vienen desarrollando los autores con el patrocinio del Instituto de Investigación Científica de la Universidad de Lima. 


\section{INTRODUCCIÓN}

\subsection{Antecedentes y problemas}

La sostenibilidad del recurso pesquero se ha visto amenazada en los últimos años debido a un sinnúmero de factores que van desde el uso de mejores tecnologías y la construcción de embarcaciones de mayor tamaño hasta la pesca por naves de bandera extranjera, el crecimiento de la población dedicada a la pesca, la falta de reglas oportunas y claras de parte del Estado y la presencia de personas que buscan obtener dinero a corto plazo, que viven del "día a día", dando lugar a la denominada "pesca negra".

Los cambios de biomasa de las especies hidrobiológicas son notables; por ejemplo, la sardina, que fue el principal recurso de la década de 1990, ha sido reemplazada por la anchoveta, especie que por sus bondades es usada para la producción de harina de pescado, que cada día es de mejor calidad. Por otro lado, también se ha incrementado su aprovechamiento para consumo humano directo o en conservas.

Otro aspecto evaluado en nuestro estudio es el cambio de reglas producido por el decreto legislativo 1084 del 18 de junio del 2008, sobre límites máximos de captura por embarcación (LMCE). La norma establece el mecanismo de ordenamiento pesquero aplicable a la extracción de anchoveta y anchoveta blanca (Engraulis ringens y Anchoa nasus) destinada al consumo humano indirecto, con el fin de mejorar las condiciones para su modernización y eficiencia; promover su desarrollo sostenido como fuente de alimentación, empleo e ingresos; y asegurar un aprovechamiento responsable de los recursos hidrobiológicos, en armonía con la preservación del medio ambiente y la conservación de la biodiversidad.

En los años recientes, con el crecimiento económico del país, el incremento de inversiones extranjeras y la necesidad de proteína en el mundo, pequeñas y medianas empresas han sido compradas por grupos empresariales que han invertido en mejorar sus tecnologías y en un aprovechamiento eficiente, disminuyendo los impactos de la industria en el medioambiente. Pero existe también la pesca artesanal, con "embarcaciones vikingas", que extraen incluso en época de veda, haciendo peligrar la sostenibilidad del recurso. 
El mayor volumen de pesca, la carencia de especies como el bonito, la caballa, el jurel y la sardina; la sobrecapacidad instalada de las plantas de producción de conservas, congelado, seco salado y los mismos productos refrigerados, han hecho que las empresas mejoren la eficiencia en el aprovechamiento de la anchoveta y la pota para la elaboración de productos para consumo humano directo. Asimismo, el empresariado pesquero está desarrollando proyectos para procesar los recursos de la acuicultura en forma sostenible.

\subsection{Justificación}

La actualización de la evolución pesquera realizada por los autores, que comprende las estadísticas sobre la disponibilidad de la biomasa, del desembarque para consumo humano directo (CHD) y para consumo humano indirecto (CHI), el estado de la flota pesquera, de la industria pesquera, de la pesca artesanal, de la cadena de comercialización en los niveles mayorista y minorista, de los terminales frigoríficos pesqueros y de los desembarcaderos pesqueros artesanales, constituyen un aporte valioso en el estudio de la sostenibilidad del recurso pesquero.

\section{METODOLOGÍA}

La investigación del tema de sostenibilidad del recurso pesquero se desarrolló, en primer término, con el respectivo estudio de gabinete, que ha comprendido, principalmente, fuentes secundarias y revisión de revistas y anuarios del sector pesquero.

En segundo lugar, para fines de contraste y validación, se realizó el trabajo de campo en el norte, centro y sur de la costa peruana, que comprendió visitas técnicas a las instalaciones de plantas pesqueras industriales y artesanales; entrevistas y conversatorios con el personal de empresas pesqueras industriales y artesanales, así como entrevistas con expertos del sector pesquero público del Instituto del Mar del Perú, Instituto Tecnológico Pesquero del Perú, Fondo Nacional de Desarrollo Pesquero y Ministerio de la Producción, con los cuales se han compartido experiencias en temas relevantes del contexto pesquero, incluyendo la sostenibilidad del producto. 


\section{RESULTADOS Y DISCUSIÓN}

\subsection{El recurso pesquero}

El recurso pesquero es aleatorio; su biomasa varía en función de varios factores: climáticos, meteorológicos, oceanográficos (fenómenos de El Niño y La Niña), sobrepesca, etcétera; los primeros factores son difíciles de predecir, a pesar de que hay estudios acerca de los ciclos que se presentan a través de los años. El factor sobrepesca sí es manejable por el hombre, aunque existen intereses geopolíticos de los países (biomasas cercanas a las fronteras), así como intereses empresariales o de pescadores por explotar al máximo posible el recurso.

Se debe tener en cuenta que el recurso pesquero es altamente perecible y que posee un elevado valor nutritivo, destacando la digeribilidad de su carne, lo que hace fácil el ataque de los microorganismos si no hay una buena conservación. Su composición química varía según la región del cuerpo y otros aspectos, como estadio sexual, tamaño, edad, lugar de captura, sexo, etcétera.

La riqueza de especies hidrobiológicas en nuestro litoral se debe a la existencia de las diversas corrientes marinas con diferentes temperaturas, grados de salinidad, oxígeno y vientos, que permiten las zonas de afloramiento y de convergencia de corrientes. En el litoral peruano existen zonas de afloramiento en Paita, Chimbote, Callao, San Juan de Marcona e Ilo, zonas ricas en recursos marinos, pues es constante el paso de aguas profundas con ricos minerales a la superficie, esencial para la producción primaria (el fitoplancton y su fotosíntesis), lo que permite la producción secundaria (zooplancton) y especies de mayor tamaño (véanse las figuras 1,2 y 3 ). 
Figura 1

Principales corrientes en el litoral peruano

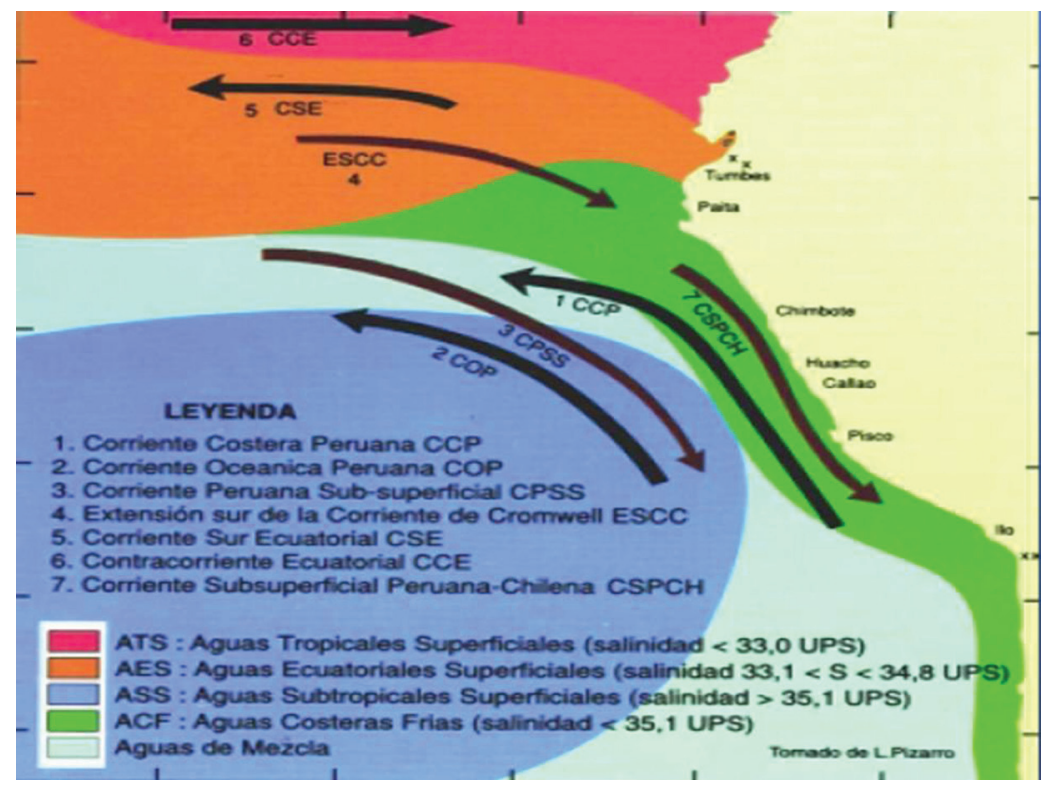

Fuente: Gil (2011).

Figura 2

Cadena trófica del ecosistema pelágico en el mar peruano

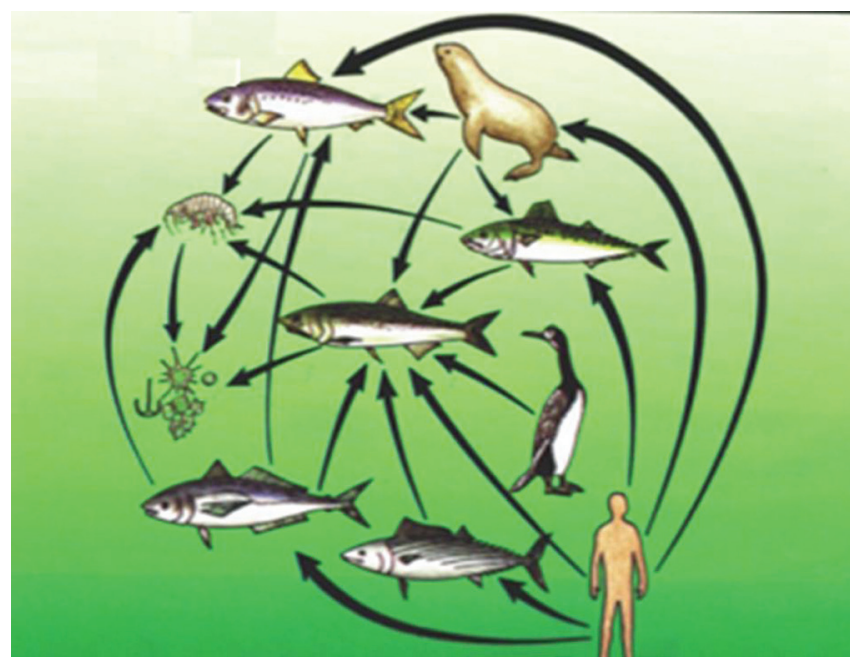

Fuente: Gil (2011). 
figura 3

Distribución de las principales especies comerciales en el ecosistema de afloramiento marino

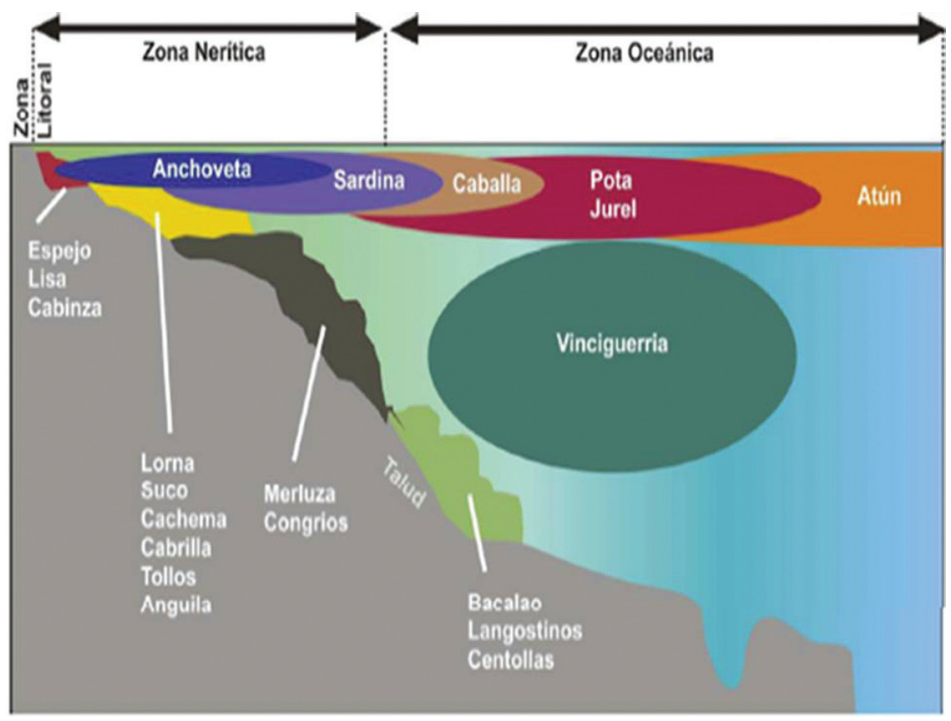

Fuente: Carbajal (2009).

El recurso pesquero debe enfriarse rápidamente y mantenerse la cadena de frío hasta el consumo final; en algunos casos es mejor conservarlo vivo, sobre todo los mariscos. Del mismo modo, es importante que se manipule adecuadamente, usando materiales y equipos limpios que permitan un producto sano, de alto valor nutritivo y de calidad.

El mar peruano es uno de los más ricos del mundo por la presencia de la corriente de Humboldt, que va de sur a norte, así como de puntos de afloramiento frente a los puertos de Ilo, San Juan de Marcona, Callao, Chimbote y Paita, que permiten una oxigenación constante de las aguas y, además, las sales minerales del ecosistema marino posibilitan una vida primaria (fitobentos y zoobentos), fitoplancton y zooplancton, filtradores y carnívoros.

Entre las especies susceptibles de explotación comercial, además de la anchoveta y el jurel, los analistas pesqueros señalan que la caballa y la pota son las de mayor potencial. 
El 2008 se aprobó el Decreto Legislativo 1084 (Ley sobre los límites máximos de permisibles de captura por embarcación), que permite el paso de la cuota de pesca global a la cuota de pesca individual. Diversos especialistas y personas vinculadas al sector opinaron que la norma haría posible el manejo de los ecosistemas y las interacciones de una pesquería sobre otra; sin embargo, los medianos y pequeños armadores que pretendían crecer se sintieron perjudicados.

Por su parte, Kisner (2009) sostiene que mientras la industria pesquera peruana posea una capacidad de captura superior a la tasa a que los ecosistemas puedan reproducirse, los recursos hidrobiológicos así como el capital no se estarán utilizando eficazmente.

En otro artículo (Kisner 2010) comenta sobre la introducción de regalías de manera que se reduzcan las inversiones artificialmente rentables. En otras palabras, si la extracción del recurso no se ajusta y se equilibra, estaremos matando la "gallina de los huevos de oro", afectando a las generaciones futuras.

Son muchos los esfuerzos de quienes nos preocupamos por salvaguardar la reserva pesquera, pero observamos que la evolución de la capacidad instalada de las embarcaciones artesanales en el Perú se ha incrementado. Algunos especialistas consideran que un 80\% de las embarcaciones son artesanales.

Parte de la solución es la pesca de altura, más aún si se tiene en cuenta la afluencia de embarcaciones extranjeras, pues los recursos son cada vez más escasos, lo cual implica que el Estado debe reglamentar la pesca de acuerdo con los tiempos que vivimos.

Todos los involucrados deberían afrontar con responsabilidad el propósito y la voluntad de gestionar mejor los recursos para las futuras generaciones.

\subsection{Captura y sostenibilidad del recurso}

En el cuadro 1 se muestra la extracción en el Perú y su relación con la pesca mundial (1979-2008). Como se puede observar, nuestro país tuvo su pico de producción más alto (10,02\%) el año 1994. 
Cuadro 1

Extracción pesquera Perú-mundo (miles de toneladas)

\begin{tabular}{|c|c|c|c|}
\hline \multirow{2}{*}{ Año } & \multirow{2}{*}{$\begin{array}{l}\text { Pesca mundial } \\
\text { y producción } \\
\text { acuícola }\end{array}$} & \multicolumn{2}{|c|}{ Pesca en el Perú } \\
\hline & & Captura & $\%$ del total \\
\hline 1979 & 64.981 & 3.653 & 5,62 \\
\hline 1980 & 65.638 & 2.709 & 4,13 \\
\hline 1981 & 68.291 & 2.717 & 3,98 \\
\hline 1982 & 70.203 & 3.514 & 5,01 \\
\hline 1983 & 71.049 & 1.570 & 2,21 \\
\hline 1984 & 77.149 & 3.318 & 4,30 \\
\hline 1985 & 79.990 & 4.138 & 5,17 \\
\hline 1986 & 85.155 & 5.562 & 6,53 \\
\hline 1987 & 88.119 & 4.584 & 5,20 \\
\hline 1988 & 104.090 & 6.637 & 6,38 \\
\hline 1989 & 105.655 & 6.852 & 6,49 \\
\hline 1990 & 102.311 & 6.842 & 6,69 \\
\hline 1991 & 102.700 & 6.914 & 6,73 \\
\hline 1992 & 107.423 & 7.564 & 7,04 \\
\hline 1993 & 111.990 & 9.098 & 8,12 \\
\hline 1994 & 120.941 & 12.118 & 10,02 \\
\hline 1995 & 124.550 & 8.971 & 7,20 \\
\hline 1996 & 128.744 & 9.487 & 7,37 \\
\hline 1997 & 128.534 & 7.838 & 6,10 \\
\hline 1998 & 123.063 & 4.310 & 3,50 \\
\hline 1999 & 132.012 & 8.392 & 6,36 \\
\hline 2000 & 138.165 & 10.626 & 7,69 \\
\hline 2001 & 135.935 & 7.956 & 5,85 \\
\hline 2002 & 139.157 & 8.741 & 6,28 \\
\hline 2003 & 139.404 & 6.061 & 4,35 \\
\hline 2004 & 148.005 & 9.574 & 6,47 \\
\hline 2005 & 150.911 & 9.353 & 6,20 \\
\hline 2006 & 152.052 & 6.984 & 4,59 \\
\hline 2007 & 155.754 & 7.179 & 4,61 \\
\hline 2008 & 159.090 & 7.363 & 4,63 \\
\hline
\end{tabular}

Fuente: Ministerio de la Producción (2009).

Elaboración propia. 
En la figura 4 se puede observar que el ciclo de la pesquería se ha ido cumpliendo periódicamente; pero en la última década este comportamiento ha sufrido una aceleración que podría deberse al efecto invernadero y la contaminación ambiental que han dado lugar a cambios oceanográficos y del clima en el planeta.

Figura 4

Fases de la pesquería

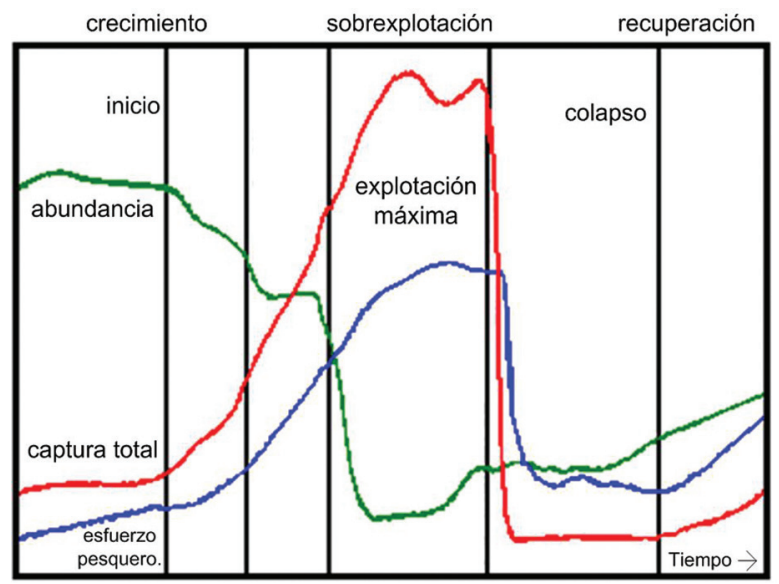

Fuente: Kleeberg y Nieto (2001).

En el cuadro 2 podemos apreciar la relación biomasa-captura realizada entre los años 1986 y 2008 para anchoveta y sardina, notándose un decrecimiento pronunciado de la sardina y un incremento de la anchoveta.

\section{Cuadro 2}

Relación biomasa-captura (miles de toneladas)

(1986-2008)

\begin{tabular}{ccccccc}
\hline & \multicolumn{3}{c}{ Anchoveta } & \multicolumn{3}{c}{ Sardina } \\
\cline { 2 - 7 } Año & Biomasa & $\begin{array}{c}\text { Captura } \\
\text { permisible }\end{array}$ & Captura & Biomasa & $\begin{array}{c}\text { Captura } \\
\text { permisible }\end{array}$ & Captura \\
\hline 1986 & 6.000 & 2.000 & 3.481 & 2.700 & 1.400 & $1.720,9$ \\
1987 & 4.500 & 1.110 & 1.764 & 3.804 & 1.900 & $2.469,2$ \\
1988 & 5.900 & 1.600 & 2.701 & 4.500 & 3.200 & $3.470,4$ \\
1989 & 4.200 & 600 & 3.720 & 3.680 & 2.200 & $2.568,9$ \\
\hline
\end{tabular}

(continúa) 
(continuación)

\begin{tabular}{|c|c|c|c|c|c|c|}
\hline \multirow[b]{2}{*}{ Año } & \multicolumn{3}{|c|}{ Anchoveta } & \multicolumn{3}{|c|}{ Sardina } \\
\hline & Biomasa & $\begin{array}{c}\text { Captura } \\
\text { permisible }\end{array}$ & Captura & Biomasa & $\begin{array}{c}\text { Captura } \\
\text { permisible }\end{array}$ & Captura \\
\hline 1990 & 5.753 & 2.300 & 2.926 & 5.748 & 2.230 & $3.205,3$ \\
\hline 1991 & 4.934 & 2.150 & 3.081 & 4.165 & 2.370 & $3.398,4$ \\
\hline 1992 & 9.033 & $\mathrm{~s} / \mathrm{d}$ & 4.870 & 5.198 & $s / r$ & $2.243,2$ \\
\hline 1993 & 11.800 & $\mathrm{~s} / \mathrm{d}$ & 7.010 & 4.029 & $s / r$ & $1.461,8$ \\
\hline 1994 & 13.500 & $s / d$ & 9.800 & 4.816 & $s / d$ & $1.551,8$ \\
\hline 1995 & 8.755 & $s / d$ & 6.558 & 4.800 & $s / d$ & $1.265,7$ \\
\hline 1996 & 7.800 & $\mathrm{~s} / \mathrm{d}$ & 7.463 & 4.400 & $\mathrm{~s} / \mathrm{d}$ & $1.056,4$ \\
\hline 1997 & 9.590 & $s / d$ & 5.928 & 2.477 & $s / d$ & 625,1 \\
\hline 1998 & 3.784 & $s / d$ & 1.206 & 2.158 & $\mathrm{~s} / \mathrm{d}$ & 908,3 \\
\hline 1999 & 5.614 & $\mathrm{~s} / \mathrm{d}$ & 6732 & $278 \mathrm{~A} /$ & $s / d$ & 187,8 \\
\hline 2000 & 4.903 & $\mathrm{~s} / \mathrm{d}$ & 9.576 & $s / d$ & $s / d$ & 226,3 \\
\hline 2001 & 11.200 & $s / d$ & 6.358 & $s / d$ & $s / d$ & 60,3 \\
\hline 2002 & 7.434 & $\mathrm{~s} / \mathrm{d}$ & 8.105 & $s / d$ & $\mathrm{~s} / \mathrm{d}$ & 6,8 \\
\hline 2003 & 7.774 & $s / d$ & 5.347 & $s / d$ & $s / d$ & 8,7 \\
\hline 2004 & 11.296 & $s / d$ & 8.808 & $\mathrm{~s} / \mathrm{d}$ & $s / d$ & 1,5 \\
\hline 2005 & 12.714 & $s / d$ & 8.655 & $\mathrm{~s} / \mathrm{d}$ & $\mathrm{s} / \mathrm{d}$ & 0,8 \\
\hline 2006 & 8.015 & $\mathrm{~s} / \mathrm{d}$ & 5.935 & $s / d$ & $s / d$ & 0 \\
\hline 2007 & 8.259 & $s / d$ & 6.160 & $s / d$ & $s / d$ & 0 \\
\hline 2008 & 9.840 & $\mathrm{~s} / \mathrm{d}$ & 6.258 & $\mathrm{~s} / \mathrm{d}$ & $\mathrm{s} / \mathrm{d}$ & 0 \\
\hline
\end{tabular}

Fuente: Ministerio de la Producción (2009).

Elaboración propia.

En el cuadro 3 se muestra el desembarque de productos marítimos para consumo humano directo entre 1970 y 2008. Estos cuadros son el resultado del análisis de los datos del Ministerio de la Producción (2009); es evidente el incremento de la pesca de la anchoveta para consumo humano.

Paredes (2010) presenta un análisis detallado de las posibles consecuencias de las últimas reformas realizadas en el sector pesquero peruano. Considera que los beneficios de la reforma son la disminución del sobredimensionamiento de la flota, la eliminación de la "carrera olímpica" (al levantarse una veda todas las flotas salían simultáneamente a pescar), la reducción en los sobrecostos y el consecuente aumento de 
Cuadro 3

Desembarque para CHD y $\mathrm{CHI}$ (miles de toneladas)

\begin{tabular}{|c|c|c|c|c|c|}
\hline \multirow{2}{*}{ Año } & \multirow{2}{*}{ Total } & \multirow{2}{*}{$\begin{array}{c}\text { Consumo humano } \\
\text { directo (CHD) }\end{array}$} & \multicolumn{3}{|c|}{$\begin{array}{l}\text { Consumo humano } \\
\text { indirecto }(\mathrm{CHI})\end{array}$} \\
\hline & & & Total & Anchoveta & $\begin{array}{c}\text { Otras } \\
\text { especies }\end{array}$ \\
\hline 1970 & $12.481,0$ & 185,3 & $12.295,7$ & $12.277,0$ & 18,7 \\
\hline 1971 & $10.505,0$ & 206,3 & $10.298,8$ & $10.276,6$ & 22,2 \\
\hline 1972 & $4.675,2$ & 213,0 & $4.462,2$ & $4.447,2$ & 15,0 \\
\hline 1973 & $2.290,0$ & 295,2 & $1.994,8$ & $1.512,8$ & 482,0 \\
\hline 1974 & $4.120,1$ & 318,9 & $3.801,2$ & $3.583,4$ & 217,8 \\
\hline 1975 & $3.409,1$ & 292,1 & $3.117,0$ & $3.078,8$ & 38,2 \\
\hline 1976 & $4.337,8$ & 333,9 & $4.003,9$ & $3.863,0$ & 140,9 \\
\hline 1977 & $2.491,4$ & 474,0 & $2.017,4$ & 792,1 & $1.225,3$ \\
\hline 1978 & $3.430,3$ & 618,8 & $2.811,5$ & $1.187,0$ & $1.624,5$ \\
\hline 1979 & $3.639,3$ & 757,6 & $2.881,7$ & $1.362,7$ & $1.519,0$ \\
\hline 1980 & $2.697,2$ & 970,0 & $1.727,2$ & 720,1 & $1.007,1$ \\
\hline 1981 & $2.700,9$ & 850,6 & $1.850,3$ & $1.225,2$ & 625,1 \\
\hline 1982 & $3.497,0$ & 551,0 & $2.946,0$ & $1.720,4$ & $1.225,6$ \\
\hline 1983 & $1.537,0$ & 309,3 & $1.227,7$ & 118,4 & $1.109,3$ \\
\hline 1984 & $3.288,4$ & 547,6 & $2.740,8$ & 23,0 & $2.717,8$ \\
\hline 1985 & $4.110,4$ & 512,5 & $3.597,9$ & 844,3 & $2.753,6$ \\
\hline 1986 & $5.529,5$ & 533,8 & $4.995,7$ & $3.481,9$ & $1.513,8$ \\
\hline 1987 & $4.548,3$ & 578,5 & $3.969,8$ & $1.764,6$ & $2.205,2$ \\
\hline 1988 & $6.598,7$ & 538,4 & $6.060,3$ & $2.701,4$ & $3.358,9$ \\
\hline 1989 & $6.818,4$ & 667,2 & 6.1512 & $3.720,2$ & $2.431,0$ \\
\hline 1990 & $6.843,2$ & 706,3 & $6.136,9$ & $2.926,4$ & $3.210,5$ \\
\hline 1991 & $6.916,1$ & 434,7 & $6.481,4$ & $3.081,0$ & $3.400,4$ \\
\hline 1992 & $7.564,5$ & 491,7 & $7.072,8$ & $4.869,9$ & $2.202,9$ \\
\hline 1993 & $9.098,3$ & 600,8 & $8.497,5$ & $7.008,5$ & $1.489,0$ \\
\hline
\end{tabular}

(continúa) 
(continuación)

Cuadro 3

Desembarque para CHD y $\mathrm{CHI}$ (miles de toneladas)

\begin{tabular}{cccccc}
\hline & & $\begin{array}{c}\text { Consumo humano } \\
\text { directo (CHD) }\end{array}$ & \multicolumn{3}{c}{$\begin{array}{c}\text { Consumo humano } \\
\text { indirecto (CHI) }\end{array}$} \\
\cline { 3 - 6 } Año & Total & Total & Total & Anchoveta & $\begin{array}{c}\text { Otras } \\
\text { especies }\end{array}$ \\
\hline 1994 & $12.118,2$ & 719,2 & $11.399,0$ & $9.799,5$ & $1.599,6$ \\
1995 & $8.970,9$ & 766,8 & $8.204,1$ & $6.557,7$ & $1.646,4$ \\
1996 & $9.486,9$ & 715,2 & $8.771,7$ & $7.460,4$ & $1.311,3$ \\
1997 & $7.837,7$ & 838,9 & $6.998,8$ & $5.923,0$ & $1.075,8$ \\
1998 & $4.310,3$ & 614,0 & $3.696,3$ & $1.205,5$ & 2490,8 \\
1999 & $8.392,4$ & 604,4 & $7.787,9$ & $6.732,0$ & $1.055,9$ \\
2000 & $10.626,3$ & 713,9 & $9.912,5$ & $9.555,6$ & 356,8 \\
2001 & $7.956,0$ & 747,9 & $7.208,0$ & $6.347,7$ & 860,4 \\
2002 & $8.741,4$ & 584,6 & $8.156,8$ & $8.082,9$ & 73,9 \\
2003 & $6.061,0$ & 714,0 & $5.347,0$ & $5.335,5$ & 11,5 \\
2004 & $9.574,3$ & 763,6 & $8.810,6$ & $8.797,1$ & 13,5 \\
2005 & $9.353,3$ & 724,6 & $8.628,7$ & $8.628,4$ & 0,3 \\
2006 & $6.983,5$ & $1.087,9$ & $5.895,5$ & $5.891,8$ & 3,7 \\
2007 & $7.178,7$ & $1.092,7$ & $6.086,0$ & $6.084,7$ & 1,3 \\
2008 & $7.363,0$ & $1.196,5$ & $6.160,7$ & $6.159,3$ & 1,4 \\
\hline & & & & & \\
\hline
\end{tabular}

Fuente: Ministerio de la Producción (2009).

Elaboración propia.

utilidades de los armadores. El citado autor opina que aún existen problemas por resolver, como la necesidad de reducir el exceso de capacidad de procesamiento de las plantas; la corrupción en el cumplimiento de la regulación; y la falta de un rol de la reforma institucional.

Como se reseñó anteriormente, la pesquería peruana ha ingresado a una nueva etapa con las cuotas de pesca de anchoveta, donde las grandes compañías pesqueras, como Tasa, Austral, Diamante, Copeinca, 
Hayduk, Exalmar y Chinese Fishery Group Investment, han comprado las medianas y pequeñas empresas y las plantas de harina se han reunificado en pocas firmas con el fin de producir harina de excelente calidad utilizando tecnología de punta. Estos grupos dominan el sector, produciendo más del $50 \%$ de harina y concentrando más del $40 \%$ de la capacidad de la flota pesquera; sin considerar su capacidad de inversión, que apunta a construir embarcaciones de 1000 toneladas de capacidad, plantas de congelado y de conservas, etcétera.

Otra arista de este tema es el incremento del número de empresas harineras dedicadas a procesar residuos de pescado, como Coprosac, Protein Fish, Pacifico Natural Foods S.A.C, entre otras, de aproximadamente $5 \mathrm{t} / \mathrm{h}$ de capacidad, que además de recibir residuos de pescado utilizan el pescado que no está en buenas condiciones de conservación por las embarcaciones de pescadores artesanales ("embarcaciones vikingas"), pues que no cuentan con sistemas de conservación a bordo, y que en algunos casos pescan dentro de las 5 millas. En junio del 2009, los pescadores artesanales denunciaron que más de cien embarcaciones de madera, conocidas como "naves vikingas", estaban pescando dentro de las 5 millas marinas, frente a las costas de Chimbote, dando lugar a la pesca negra, que es muy perjudicial para la sostenibilidad del recurso.

En lo relativo al precio de la harina de pescado, este es muy competitivo y rentable para los empresarios. Es importante recordar también la preocupación que ha tenido el Estado en incentivar la elaboración de productos para consumo humano directo, a partir de la anchoveta; aunque esto no ha sido fácil debido a la idiosincrasia del poblador peruano, que no consume esta especie por considerar solo como un insumo para la elaboración de harina de pescado.

La materia prima para la producción de harina de pescado es cada vez más escasa y costosa, y sus precios son cada vez mayores; por ejemplo, la tonelada de anchoveta ha llegado a la cifra de 300 dólares, lo que ha dado lugar a que la harina super prime haya alcanzado precios cercanos a los 2.000 dólares la tonelada. Este aumento también ha sido consecuencia del desastre causado por el terremoto en Chile, que asoló el país del sur. Muchos expertos han intentado sustituir la harina de pescado por la de soya en la alimentación de peces, por ser sostenible y de menor precio en el mercado; pero cada día son más las propiedades que encuentran en la harina de pescado que la hace difícil de sustituir. 
La pota es otra especie que adquiere importancia en la elaboración de productos congelados y envasados con valor agregado, como hamburguesas, snacks, etcétera. Cuando se obtienen grandes volúmenes de esta especie, algunas empresas los usan para elaborar harina de pescado.

En el cuadro 4, se presentan los volúmnes de utilización de la anchoveta para consumo humano directo e indirecto, como harina y aceite de pescado. Se puede observar que en el 2009 el incremento de la producción de conservas de anchoveta comercializadas en el mercado nacional fue significativo.

\section{Cuadro 4}

Uso de anchoveta para $\mathrm{CHD}$ y $\mathrm{CHI}$ (harina) (miles de toneladas)

\begin{tabular}{lrrrrr}
\hline Año & \multicolumn{1}{c}{$\mathbf{2 0 0 5}$} & \multicolumn{1}{c}{$\mathbf{2 0 0 6}$} & \multicolumn{1}{c}{$\mathbf{2 0 0 7}$} & \multicolumn{1}{c}{$\mathbf{2 0 0 8}$} & \multicolumn{1}{c}{$\mathbf{2 0 0 9}$} \\
\hline Harina total & $8.628,00$ & $5.891,40$ & $6.071,9$ & $6.116,3$ & $5.823,2$ \\
CHD total & 27,07 & 43,46 & 71,17 & 90,43 & $1.130,3$ \\
Fresco & 0,35 & 0,54 & 0,57 & 0,31 & 411,3 \\
Enlatado & 14,89 & 31,00 & 58,12 & 77,07 & 163,9 \\
Congelado & 1,41 & 1,27 & 5,18 & 8,42 & 44,8 \\
Curado & 10,43 & 10,66 & 7,30 & 4,63 & 510,3 \\
\hline
\end{tabular}

Fuente: Ministerio de la Producción (2010).

Elaboración propia.

\subsection{La producción y la sostenibilidad del recurso}

Como lo demuestran las estadísticas de las exportaciones, el sector pesquero sigue siendo uno de los más importantes de la economía peruana. El incremento del precio de la harina y del aceite de pescado ha sido constante entre el 2005 y el 2009, pasando en promedio anual de 560 a 900 dólares la tonelada. Entre los últimos meses del 2009 y agosto del 2010, este precio ha tenido su más alta cotización, al duplicarse prácticamente el precio promedio del 2009. En el cuadro 5 se muestra la participación del sector pesquero en el total de exportaciones.

El proceso de elaboración de harina y aceite de pescado es intensivo en el uso de maquinaria, por lo que los principales costos del sector, además de la materia prima, lo constituyen la energía utilizada en el proceso productivo, así como el mantenimiento y la inversión en maquinaria. 
Por otro lado, es importante señalar que existen tres tipos genéricos de harina de pescado: FAQ o estándar, prime y súper prime, las que varían de precio según la proporción de proteínas y nutrientes en general. En el Perú, en la década de 1990, cerca del 60\% de la producción harinera corresponde a $\mathrm{FAQ}$, un $35 \%$ a prime y solo un $5 \%$ a súper prime. En los últimos años (2010) esta relación ha variado, como consecuencia de la modernización de las plantas y los buenos precios de las harinas de mejor calidad, como la prime y súper prime.

\begin{tabular}{|c|c|c|c|}
\hline Años & $\begin{array}{l}\text { Exportación total } \\
\text { (US\$) }\end{array}$ & $\begin{array}{c}\text { Exportación pesca } \\
\text { (US\$) }\end{array}$ & $\%$ \\
\hline 1998 & 5.725 .840 & 638.550 & 11,15 \\
\hline 1999 & 6.040 .340 & 790.980 & 13,09 \\
\hline 2000 & 6.881 .350 & 1.141 .160 & 16,58 \\
\hline 2001 & 6.956 .250 & 1.133 .870 & 16,30 \\
\hline 2002 & 7.665 .260 & 1.066 .920 & 13,92 \\
\hline 2003 & 9.026 .640 & 1.031 .490 & 11,43 \\
\hline 2004 & 12.778 .230 & 1.387 .620 & 10,86 \\
\hline 2005 & 17.299 .920 & 1.634 .440 & 9,45 \\
\hline 2006 & 23.799 .890 & 1.775 .410 & 7,46 \\
\hline 2007 & 27.800 .070 & 1.962 .820 & 7,06 \\
\hline 2008 & 31.162 .750 & 2.417 .470 & 7,76 \\
\hline
\end{tabular}

Fuente: Ministerio de la Producción (2009).

Elaboración propia.

Fuentes del Ministerio de la Producción han destacado que desde que se implementó el sistema de cuotas de pesca, la producción de harina de pescado tipo súper prime, que es la de más alta calidad en los mercados internacionales, se ha incrementado y ahora representa el $12 \%$ del total. Entre tanto, la producción de la harina de pescado tipo prime también ha aumentado hasta representar el 50\% del total mientras que la producción de harina tradicional ha reducido su participación en la fabricación de harina de pescado nacional y ahora solo representa el $38 \%$. 
Todo ello redunda en un mejor manejo de la producción; se evita la pérdidas innecesarias de los empresarios en las embarcaciones, el combustible y los recursos humanos, mejorando su eficiencia y la calidad de la materia prima, pescando lo justo y necesario en el momento y lugar adecuados. Así, también, redundará en un mejor cuidado del medioambiente y una mejor calidad de vida del pescador. La intención de las principales empresas es la de reconvertir las plantas harineras para producir una mayor cantidad de harina súper prime, donde los márgenes de ganancia son mayores.

\subsection{La acuicultura y la sostenibilidad del recurso}

La producción de alimentos procedentes de la acuicultura de aguas marinas y continentales está creciendo de manera acelerada en todo el mundo en los últimos años; así, se ha convertido en una importante fuente de empleo, ingresos y suministro de alimentos, que contribuye a la seguridad alimentaria en el mundo. Su ritmo de crecimiento ha sido mayor que el de otros sectores de la producción con materias primas de origen animal. Mientras la producción de la pesca de captura cesó de crecer a mediados de la década de 1980, el sector acuícola ha mantenido una tasa de crecimiento medio anual en el mundo de $8,7 \%$.

Actualmente, la acuicultura en el Perú se orienta a la producción de langostino (Litopenaeus vannamei), concha de abanico (Argopecten purpuratus), trucha (Oncorhynchus mykiss) y tilapia (Oreochromis spp.), entre los principales. Asimismo, la acuicultura viene emergiendo como un rubro de producción económica importante por las condiciones que ofrece el territorio peruano en cuanto al clima y extensión de los espejos de agua propicios para esta actividad, que alcanzó poco más de 43 mil toneladas en el 2009. En el cuadro 6 se presenta la cosecha de recursos hidrobiológicos procedentes de la acuicultura según ámbito y especie.

Se puede apreciar que todos los sistemas de producción acuícola experimentaron un crecimiento notable a partir del 2002, particularmente los cultivos industriales de concha de abanico, langostinos y truchas, aunque la producción de tilapia y de peces amazónicos también creció durante este periodo, pero en una menor proporción. Cabe señalar que en el año 2008, el cultivo de concha de abanico bajó debido a eventos naturales como la marea roja y el ingreso de corrientes cálidas que afectaron con altas mortalidades el cultivo y la producción de semilla 


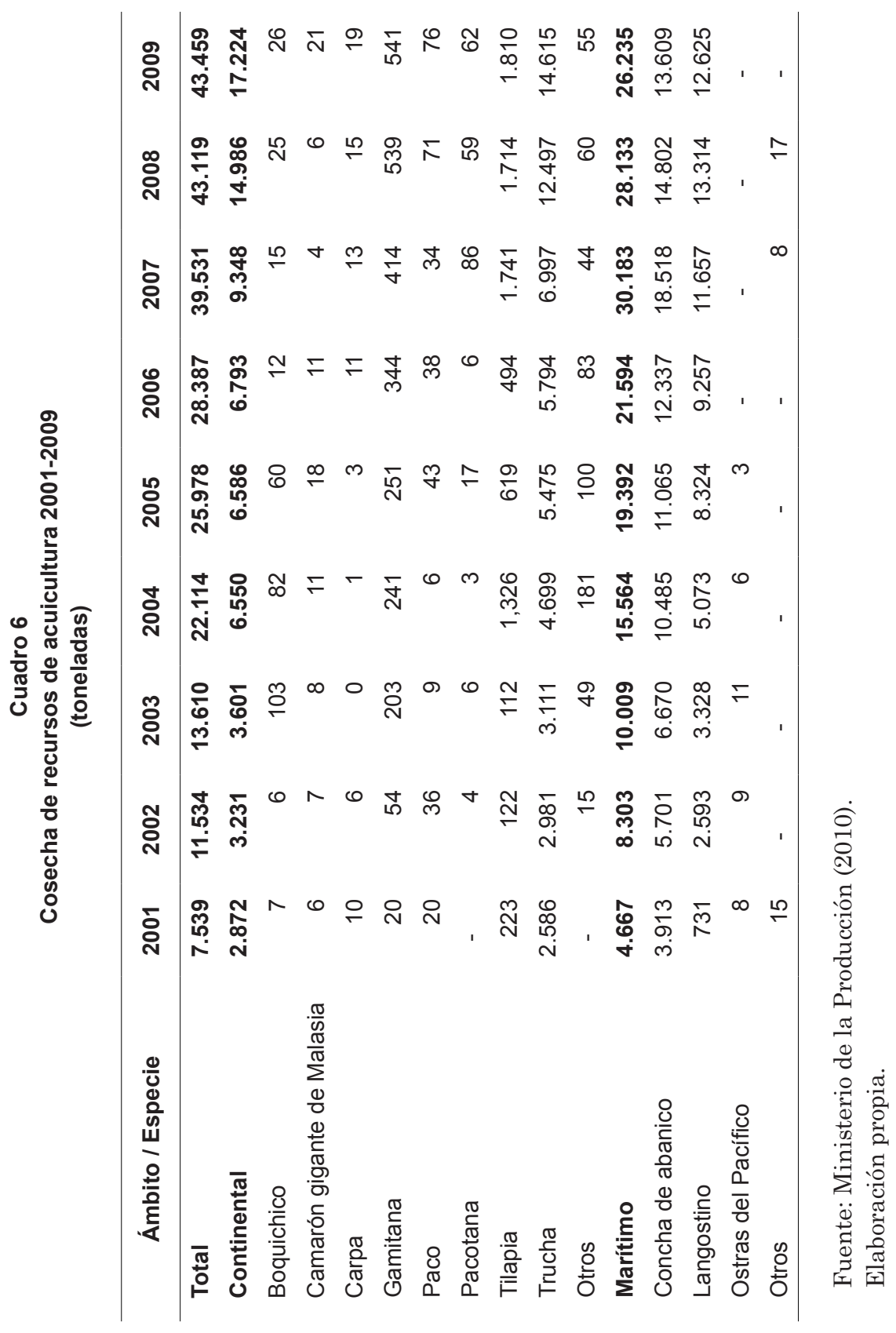


de dicha especie. Es interesante revisar el desarrollo de la acuicultura en Piura, en especial el caso del langostino cultivado con aguas del río Chira por la empresa EcoAcuícola SAC, que, cumpliendo con todas las certificaciones, está cultivando langostino en agua dulce con muy buenos resultados. Se compran las semillas del Ecuador y se dispone de tecnología para la eclosión, cultivo y engorde del langostino hasta que alcance su peso comercial de 24 gramos.

Por otro lado, en Parachique, Sechura, donde los pescadores prácticamente han parcelado el mar, existe un gran movimiento económico basado en la concha de abanico, con una descarga diaria de grandes volúmenes. Esta gran bahía, la más grande del Perú, presenta una importante productividad, sobre todo de la concha de abanico, su principal recurso hidrobiológico, debido a lo cual el Ministerio de la Producción regula las concesiones de maricultura en la bahía. En esta zona, las organizaciones sociales de pescadores artesanales, conocidas como OSPA, recogen la concha de los núcleos o de la zona denominada isla Lobos de Tierra, y la echan en sus corrales o simplemente en los límites de sus concesiones, un sistema considerado como repoblamiento del recurso en la zona. La cantidad de poslarvas de la concha que se capta bastaría para que se pueda sembrar luego de su desarrollo, al cabo de un mes o dos, dependiendo de las condiciones oceanográficas y otros factores, para repoblar el fondo marino y así dejar tranquila la isla Lobos de Tierra; asimismo, se podrían disminuir los costos de ir hasta tan lejana isla. Con la formalización de 23 asociaciones y otras 4 autorizadas, se ha ordenado el $30 \%$ de la bahía de Sechura en cumplimiento de las demandas del Servicio Nacional de Sanidad Pesquera (Sanipes) del Instituto Tecnológico Pesquero (ITP), ante las exigencias que están imponiendo los mercados europeos para la exportación del recurso concha de abanico.

Todos estos avances deben ir de la mano de un adecuado manejo y control ambiental en tierra. Se sabe que el ITP se preocupa por la sanidad e higiene, pero el problema es el agua y su manejo, su reciclaje y sus altos costos. Es un reto que debemos afrontar.

La publicación digital Aguahoy (2010) nos informa que los principales productos de la acuicultura peruana se han exportado por US $\$ 142$ millones en el primer semestre del 2010, un 39\% más que en similar periodo del año anterior, cuando el monto ascendió a US\$102 millones, según informó la Gerencia de Industrias Extractivas de la Asociación 
de Exportadores (ADEX), al tiempo de destacar el potencial de ese rubro, que despierta gran interés en empresarios de otros sectores, como el minero y el agrario.

Efectivamente, empresas mineras apoyan esa actividad en las comunidades aledañas a sus operaciones, y empresas del sector agrario también hacen lo propio, como el caso de Camposol, empresa ligada al agro, que anunció la compra de las compañías langostineras Domingo Rodas S.A. y Camarones S.A., además de 170 hectáreas adicionales. Según cifras del área de Inteligencia Comercial de ADEX, la principal partida de nuestra acuicultura es la que corresponde al ítem "Demás moluscos e invertebrados acuáticos", con envíos, el 2010, por US\$55,40 millones, que representan 66,7\% más que entre enero y junio del año anterior (US\$33 millones). En esta partida se exporta pota en sus diversas presentaciones: congelada, picada, cocida, fileteada, entre otros.

La segunda partida es "Veneras (vieiras y conchas de abanico) congeladas, secas, saladas o en salmuera”, que creció en $67 \%$, al registrar envíos por US\$35 millones, mientras que la exportación de "colas de langostinos sin cocer en agua o vapor" descendió en 4\% (US\$24 millones).

Respecto de los destinos, se debe señalar que en el caso de la partida "Demás moluscos e invertebrados acuáticos", el principal comprador es China, que incrementó su demanda en 115\% (US\$40,9 millones), concentrando de esa manera el 74\% del total de las exportaciones; le siguen Corea del Sur, Estados Unidos, Alemania, Japón, España, Italia y Taiwán, entre otros.

La partida "Veneras (vieiras y conchas de abanico) congeladas, secas, saladas o en salmuera" tiene como principal destino Francia (US\$19 millones), seguida de Países Bajos, Estados Unidos, Bélgica, Italia, España, Reino Unido, Chile, Portugal y Canadá, entre otros. La partida "colas de langostino sin cocer en agua o vapor, congeladas" se envía a Estados Unidos (US\$22,6 millones), España (US\$625 mil), Ecuador, Bélgica, Lituania y Rusia.

Otras partidas de la acuicultura peruana son "langostinos enteros congelados", "harina de crustáceos y otros invertebrados", "colas de langostinos sin caparazón congeladas", "peces ornamentales", "almejas y machas" y "truchas congeladas". En el cuadro 7 se presenta la exportación de especies cultivadas en el Perú. 


\section{Cuadro 7}

Exportación de especies cultivadas en el Perú (toneladas)

\begin{tabular}{lcccccccc}
\hline \multirow{2}{*}{ Especies } & \multicolumn{8}{c}{ Años } \\
\cline { 2 - 9 } & $\mathbf{2 0 0 1}$ & $\mathbf{2 0 0 2}$ & $\mathbf{2 0 0 3}$ & $\mathbf{2 0 0 4}$ & $\mathbf{2 0 0 5}$ & $\mathbf{2 0 0 6}$ & $\mathbf{2 0 0 7}$ & $\mathbf{2 0 0 8}$ \\
\hline Langostino & 554 & 1.331 & 2.925 & 3.658 & 6.150 & 7.555 & 9.023 & 3.527 \\
$\begin{array}{l}\text { Concha de } \\
\text { abanico }\end{array}$ & 601 & 604 & 1.418 & 2.103 & 2.408 & 2.289 & 3.131 & 1.139 \\
Trucha & 276 & 361 & 441 & 422 & 754 & 857 & 795 & 155 \\
Tilapia & 108 & - & - & 155 & 40 & 0 & 8 & 0 \\
Total & 1.539 & 2.296 & 4.784 & 6.338 & 9.352 & 10.701 & 12.958 & 4.820 \\
\hline
\end{tabular}

Fuente: Ministerio de la Producción (2009).

Elaboración propia.

\section{CONCLUSIONES}

La industria pesquera ha cambiado en los últimos años. Un factor muy importante son las cuotas de pesca, que ha ordenado el panorama pesquero. Asimismo, se deben considerar los altos precios de la harina de pescado como consecuencia de la escasez de materia prima, la demanda de mayor proteína de alto valor biológico y el crecimiento de la acuicultura, que han dado lugar a que la harina llegue a precios cercanos a los dos mil dólares la tonelada, y la anchoveta, especie con características muy apreciadas por sus consumidores, a 300 dólares la tonelada, lo que hace que las harinas especiales sean productos más competitivos que los productos de consumo humano directo.

La mayoría de investigadores consideran entre los aspectos positivos de la asignación de cuotas individuales de captura el aumento del número de días de pesca; esto permitió planificar la actividad extractiva y mejorar la calidad de los desembarques y de la harina, además de reducir la congestión y la contaminación de las bahías. Se redujo el número de embarcaciones activas, con lo que disminuyeron los costos y mejoraron las utilidades.

Según León (2010), en su artículo "Informe. La segunda ola", se resume lo que pasa en el sector pesquero de nuestros días. La fuerte alza que experimentó el precio de la anchoveta en los últimos años 
ha llevado a los principales grupos pesqueros a salir nuevamente de compras. Todos quieren crecer y seguir captando una mayor cuota. Al 2010, según el referido informe, las principales pesqueras y sus respectivos grados de participación en las cuotas de pesca son: TASA (14\%), Copeinca (10,8\%), Diamante (8,11\%), Austral $(6,98 \%)$, Hayduk $(6,68)$, Exalmar (5,47), CFG Investment (6,05), Grupo Romero (2,18\%). Otras empresas independientes que trabajan con embarcaciones de acero y de madera tienen el $32 \%$ de la pesca total del país. Concluye su análisis destacando el aporte de funcionarios consultores de Interbank, que consideran que la nueva ola de compras podría ser relativamente larga, porque muchas compañías pequeñas se resisten a vender y porque la disputa esta vez es de barco a barco y punto por punto de cuota asignada a los armadores, que tienen en sus manos el $12 \%$ de la cuota global de pesca.

Para Kisner (2010) la abundancia de anchoveta en el Perú y su rentable conversión en harina han sido un freno para el desarrollo de una cultura de la alimentación basada en el primer recurso con el que contamos los peruanos: el pescado, rico en proteínas, grasa y fósforo; un alimento de primera calidad fundamental para la buena nutrición infantil, cuya abundancia ha resultado más una tragedia que un beneficio, concluye.

Antes las plantas conserveras trabajaban principalmente con sardina, pero hoy esta especie escasea, por lo que se trabaja con caballa, jurel y anchoveta, esta última cada vez con mayor participación. Los precios de las conservas de atún o bonito se han vuelto inalcanzables para las mesas populares, que las han sustituido por conservas de anchoveta, que el Estado debe seguir promoviendo mediante una política que incentive su consumo. Esto se logrará con el ordenamiento de la pesca artesanal y evitando que en época de veda se siga procesando harina de pescado entero en las plantas de harina de residuos y con una educación constante que ponga énfasis en que la anchoveta es un recurso de alto valor nutritivo y agradable sabor.

Por último, para el desarrollo de la acuicultura es indispensable un buen manejo del recurso hídrico que cada día será más escaso y costoso. 


\section{BIBLIOGRAFÍA}

Aquahoy (agosto del 2010). "Perú: Productos de la acuicultura peruana se exportaron por 142 millones de dólares". Aquahoy [portal de noticias]. <http://www.aquahoy.com>.

Carbajal, V. W. (2009). "Perspectivas de la actividad pesquera en el norte del Perú". Ponencia presentada en el fórum "El sector pesquero en la región Piura: Situación actual y perspectivas", organizado por el Instituto del Mar del Perú (Imarpe) y la Municipalidad Provincial de Paita. Paita, 25 de junio.

Diario oficial El Peruano (2008). Normas legales. Decreto Legislativo 1084.

Gil, K. P. (2011). Recursos pesqueros en el Perú. Lima: Universidad Nacional Agraria.

Gracia, G. A. (1994). "El manejo fino de pesquerías: La transición hacia un manejo equilibrado y sustentable de los recursos". Industria Alimentaria 1. Vol. 16.2.

Kisner, B. M. (11 de noviembre del 2010). "La pesquería del jurel en el Océano Pacífico suroriental". Revista Pesca. Lima.

——. (8 de agosto del 2008). "El modelo de la gestión pesquera". Revista Pesca. Lima.

Kleeberg, H. F. y D. M. Rojas (2010). "La industria pesquera en el Perú". Proyecto de investigación. Lima: Universidad de Lima, IDIC.

Kleeberg, H. F y V. M. Nieto (2001). La industria pesquera en el Perú. Lima: Universidad de Lima, Fondo Editorial.

León, A. (25 de octubre del 2010). "El informe: La segunda ola". El Comercio. Lima.

Ministerio de la Producción (2009). Datos estadísticos del sector pesquero. Lima: Produce.

Paredes, C. E. (2010). "Reformando el sector de la anchoveta peruana, progreso reciente y desafíos futuros". Ponencia presentada en la conferencia organizada por el Instituto del Perú. Lima, 7 de julio. 